\title{
WHICH CHORD PROGRESSIONS SATISFY US THE MOST? THE EFFECT OF EXPECTANCY, MUSIC EDUCATION, AND PITCH HEIGHT
}

\author{
Žiga Mekiš Recek*, Zala Rojs, Laura Šinkovec, Petra Štibelj, \\ Martin Vogrin, Brina Zamrnik and Anka Slana Ozimič
}

University of Ljubljana, Faculty of Arts, Department of Psychology

Ljubljana, Slovenia

DOI: 10.7906/indecs.19.4.9

Received: 25 January 2021.

Regular article

Accepted: 21 May 2021.

\begin{abstract}
Music is an integral part of our everyday lives. Through continuous exposure to a particular music style, an individual implicitly learns the laws of music, including the typical progression of chords that accompany the leading melody. Previous research has shown that the typical chord order in compositions is perceived as expected and satisfying, whereas the violations of the typical chord progressions are perceived as unexpected and unsatisfying. In this paper, we investigated how implicit musical knowledge influences satisfaction during listening to expected and unexpected chord progressions by taking into account the participant's music education and the overall pitch height of the chordal sequences. Ninety-seven participants (43 musicians and 54 non-musicians) took part in the experiment. They were asked to rate the degree of their satisfaction during listening to expected and unexpected chord progressions, either during the high-pitch or low-pitch height conditions. The results showed that the participants were more satisfied with expected than unexpected chord progressions, confirming previous findings on the role of implicit learning of rules of harmony. Although results did not reveal an effect of music education during listening to expected chord progressions, musicians evaluated unexpected progressions as less satisfying than non-musicians, suggesting that musicians' are more susceptible to violations of typical chord order. Finally, the results have shown that the difference in satisfaction between expected and unexpected progressions was larger in high-pitch vs. low-pitch condition, suggesting that under low-pitch condition, chord progressions were more difficult to discriminate, confirming the theory of low-interval limit.
\end{abstract}

\section{KEY WORDS}

harmonic progression, implicit learning, low interval limit, music education, satisfaction

\section{CLASSIFICATION}

APA: $2300,2326,2340,2343,2360$

JEL: $\quad$ I39 


\section{INTRODUCTION}

Music is an integral part of our everyday lives. Through exposure to a particular music style, an individual implicitly learns the laws of rhythm, melody, harmony, and other aspects of sound organization, forming implicit expectations about how these features are supposed to develop through auditory experience [1]. If these expectations are met, the individual experiences satisfaction while listening to music and vice versa - if the expectations are violated, the listener is not satisfied with the listening experience [2]. The aim of this paper was to investigate how implicit musical knowledge influences satisfaction during listening to expected and unexpected harmonic musical sequences and how this interacts with the participant's music education and the overall pitch height of the sequences.

Music is formed by a series of notes played sequentially or simultaneously, forming a melody and harmony, respectively. A chord, which is the fundamental building block of harmony, consists of three or more simultaneously played notes, while a chord progression is a sequence of chords. The most common chord order in traditional western music is a tonic - tonic in the first inversion-subdominant-dominant-tonic [3]. Specifically, a typical chord progression begins and ends with a tonic chord, whose keynote matches the scale. For example, in a composition that is written in $\mathrm{C}$ major, the keynote is $\mathrm{C}$, and the tonic chord consists of C, E, and G. After the first tonic chord, inverted tonic chords, whose function is to lengthen the sense of the first tonic chord, usually follow. An inverted chord simply means that a note other than the keynote is at the bottom. Based on the lowest note from the chord (e.g., $\mathrm{E}$ or $\mathrm{G}$ in $\mathrm{C}$ major), we can determine its inversion. In the first inversion, the lowest note is the third of the triad ( $\mathrm{E}$ in $\mathrm{C}$ major), with the fifth ( $\mathrm{G}$ in $\mathrm{C}$ major) and the keynote ( $\mathrm{C}$ in $\mathrm{C}$ major) stacked above it. After the lengthening of the tonic chord with the inverted tonic chords, the subdominant (e.g., in $\mathrm{C}$ major, the subdominant chord consists of $\mathrm{F}, \mathrm{A}, \mathrm{C}$ ) and the dominant (e.g., in $\mathrm{C}$ major, the dominant chord consists of G, B, D) usually follow. Same as at the start of the progression, the chord progression typically ends with the tonic.

With the continuous exposure to western music, individuals implicitly internalize the structure of the typical chord progression to the extent that the typical form of the harmonic syntax in compositions is perceived as expected and satisfying, whereas the violations of the syntax rules are perceived as unexpected and unsatisfying [2, 4]. Loui and Wessel [5] have demonstrated this effect for both musicians and non-musicians. Although musicians were, in general, more satisfied when listening to musical sequences than non-musicians, the pattern of satisfaction to expected and unexpected chord progressions did not differ between musicians and non-musicians [5]. Similarly, the sensitivity to the violations of the typical harmonic context of both musicians and non-musicians has been shown by reaction times $[6,7]$ and event-related potentials $[8,9]$ studies. Together these studies show that both musicians and non-musicians implicitly internalize rules of music harmony and make harmonic expectations, on which they base their response for a musical phrase.

In contrast to Loui and Wessel [5], Dellacherie et al. [10] found differences between musicians and non-musicians in how they respond to expected and unexpected musical sequences. Specifically, they investigated the response to consonant and dissonant musical excerpts. Dissonances, which can be defined as a combination of sounds that do not belong to a particular musical style [11], can - similarly to the unexpected chord resolutions - be treated as tensions in western music, which can induce unexpectancy and unpleasantness during listening to music. Dellacherie et al. [10] found greater negative emotional self-reports and physiological response of musicians to dissonances compared to non-musicians, whereas their response to consonant excerpts did not differ. They explain their findings with the possibility that a negative bias is formed through the experience of music training. They 
proposed that because of long-term associative learning, a link between dissonance and negative emotions is formed. Consistent with Dellacherie et al. [10], Pagès-Portabella and Toro [12] reported that during listening to dissonances, early right anterior negativity as measured by EEG was observed in both musicians and non-musicians, indicating automatic processing of harmonic inconsistencies (i.e., violations of musical syntax). However, a larger effect of harmonic inconsistencies was reported for musicians vs. non-musicians, suggesting that music training modulates how different violations of the harmonic context are processed.

Since musical education seems to modulate the response to violations of music syntax, another intriguing question is whether the overall pitch height of chord progression also plays a modulatory role. Pitch height seems to be an important factor that composers consider when creating music. In order to achieve clarity and purity between the intervals, the tones are presented above the so-called 'low interval limit', a concept that determines the lowest pitches at which intervals can still be perceived without sounding uncollected or muddy [13]. When the lower tones become too layered, they create dissonance and lose their clarity [14]. The limits are not absolute but represent areas below which there is a risk that the resultant sound will not work well within a normal harmonic context. To our knowledge, no study so far has investigated how pitch height influences satisfaction while listening to expected and unexpected chord progressions. Based on the low interval limit concept, we hypothesize that resolutions of chord progressions in the low-pitch condition will be experienced as unfocused, which may prevent participants from discriminating between the types of resolutions. This should result in a smaller difference in satisfaction between expected and unexpected chord progressions in low- vs. high-pitch conditions.

In summary, our study aimed to investigate how implicit musical knowledge affects satisfaction while listening to expected and unexpected resolutions of chord progressions and how this interacts with music education and the overall pitch height of chord progressions. Because of the internalization of the typical harmonic syntax $[7,8,15]$, we assume that both musicians and non-musicians will experience chord progressions that are frequently used and are typical in western music as more satisfying than uncommon chord progressions. Furthermore, based on Loui and Wessel's [5] study, we expected that the overall satisfaction for both expected and unexpected chord progressions would be higher in musicians vs. non-musicians. As studies investigating the effect of musical education on the perception of violations of musical syntax do not yield consistent findings (e.g. [5, 10, 12]), two alternative hypotheses can be formed. First, we might expect that the effect of expectancy will be larger in musicians vs. non-musicians, as their extensive exposure to music might lead to even stronger internalization of musical syntax [10]. The alternative is that the effect of expectancy will not differ between musicians and non-musicians, which would indicate that both groups implicitly learn the harmony to a similar extent and that this is independent of music education [5]. Lastly, we were interested in the effect of pitch height of the chord progressions. As explained above, based on the low-interval limit concept, we expect the effect of expectancy to be larger in high vs. low-pitch positions, as in lower pitch positions, chords might be perceived as unclear and dissonant [14], making it more difficult to discriminate between expected and unexpected resolutions and thus reducing the effect of expectancy.

\section{METHOD}

\section{PARTICIPANTS}

194 Slovenian university and high school students, including students of music conservatories, signed the informed consent to participate in an online experiment. The results of 89 participants who did not complete the experimental task were excluded from the analysis. 
Further, the results of another 8 participants were excluded from the analysis because they completed the task in less than 10 minutes, which was estimated to be the minimal time needed to perform the task. The final sample included 97 participants ( 71 females), aged from 15 to 25 years $(M=18,37, S D=2,07)$. Those with more than eight years of formal education in music theory ( $N=43$ participants) were considered musicians, while the participants with less than eight years of formal education in the field of music theory were considered nonmusicians $(N=54$ participants).

\section{APPARATUS AND MATERIALS}

Nine typical chord progressions that consisted of five chords (Table 1, Figure 1) were constructed for the experiment. They either had an expected (the progression resolved on the I. chord or the tonic of the key) or unexpected (the progression resolved on the IV. chord or the subdominant of the key) resolution.

Table 1. List of chord progressions with an expected and an unexpected resolution.

\begin{tabular}{|l|l|}
\hline \multicolumn{1}{|c|}{ Expected resolution } & \multicolumn{1}{c|}{ Unexpected resolution } \\
\hline G-C-F-G-C (V-I-IV-V-I) & G-C-F-G-F (V-I-IV-V-IV) \\
\hline C-a-F-G-C (I-vi-IV-V-I) & C-a-F-G-F (I-vi-IV-V-IV) \\
\hline e-a-d-G-C (iii-vi-ii-V-I) & e-a-d-G-F (iii-vi-ii-V-IV) \\
\hline C-a-d-G-C (I-vi-ii-V-I) & C-a-d-G-F (I-vi-ii-V-IV) \\
\hline C-G-a-F-C (I-V-vi-IV-I) & C-G-a-F-F (I-V-vi-IV-IV) \\
\hline C-F-a-G-C (I-IV-vi-V-I) & C-F-a-G-F (I-IV-vi-V-IV) \\
\hline C-e-F-G-C (I-iii-IV-V-I) & C-e-F-G-F (I-iii-IV-V-IV) \\
\hline C-F-C-G-C (I-IV-I-V-I) & C-F-C-G-F (I-IV-I-V-IV) \\
\hline C-F-d-G-C (I-IV-ii-V-I) & C-F-d-G-F (I-IV-ii-V-IV) \\
\hline
\end{tabular}

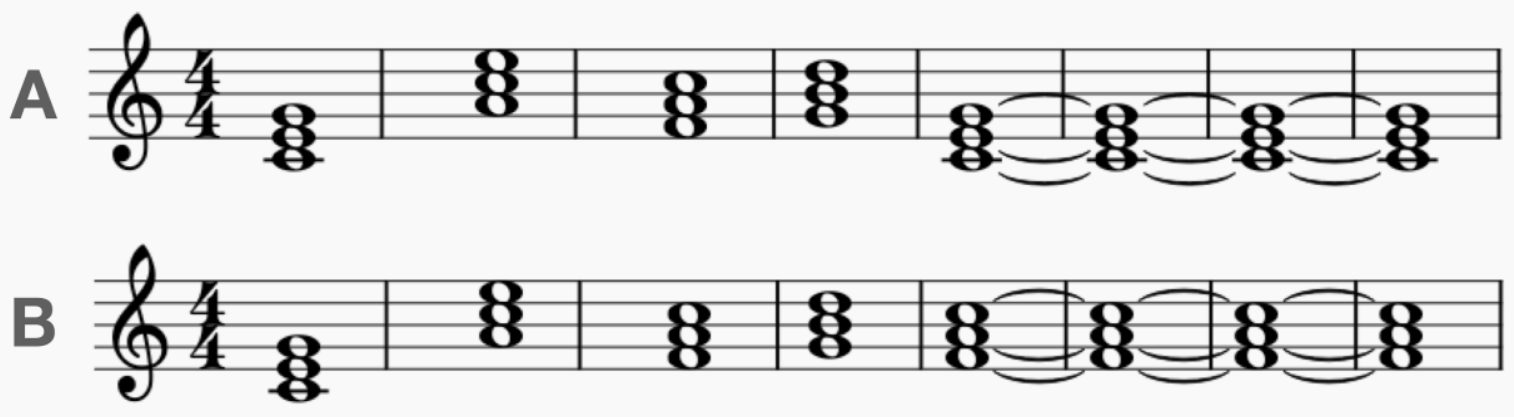

Figure 1. Chord progression examples. A) Chord progression with an expected resolution (the progression ends on the I. chord). B) Chord progression with an unexpected resolution (the progression ends on the IV. chord).

The constructed expected and unexpected chord progressions were recorded in low and high pitch conditions. In low pitch condition, the tonic of the chord progression was note $\mathrm{C} 2(65,41 \mathrm{~Hz})$, and in high pitch condition, the tonic of the progression was note $\mathrm{C} 4(262 \mathrm{~Hz})$. Each of the 
constructed chord progressions was additionally transposed by or two semitones higher or lower than the original progression, resulting in two starting points for each chord progression (original, transposed).

All progressions were recorded in the time signature of $4 / 4$ in the tempo of 130 beats per minute within eight bars. All of the chords were played within one bar, except for the last, resolving chord, which was played over the last four bars. The progressions were recorded digitally using Logic Pro X, ver. 10.4.4. [16], which is a digital audio workstation. We used a virtual sampled upright piano called 'The Gentleman' [17] that runs in Kontakt 6 player [18] - an industry-standard software for sampling. This provided a clean, clear, and natural sound of a piano with a touch of natural reverb. Psytoolkit software [19, 20] was used to present the chord progressions and collect keyboard-click responses.

\section{TASK}

The experimental task consisted of 72 trials ( 9 chord progressions $\times 2$ resolution types $\times 2$ pitch heights $\times 2$ starting points) presented in random order across participants. Each trial started with the presentation of the chord progression lasting for about $15 \mathrm{~s}$. Following the chord progression, the participant's task was to rate each progression on a scale from 0 (not satisfactory at all) to 100 (most satisfactory) depending on how much subjective satisfaction they experienced while listening to it.

\section{PROCEDURE}

We used the snowball sampling method to reach the participants. Participants were informed about the study through different web mediums, such as e-mail, Facebook posts, direct messages, etc. Those interested in participating in the study accessed the study through an experimental website, which contained a detailed description of the study's purpose, terms, and the task itself.

Before signing the informed consent, the participants were instructed to perform the task in a quiet environment using headphones, which allowed them to attend to the task without any interruption and to take a break if they felt fatigued. They were able to test whether both left and right channels of their headphones work properly.

After reading and signing the informed consent, the participants were asked to provide information regarding their age and musical education. After they have read the instructions, they could begin the experimental task. At the end of the experiment, they could provide comments regarding their experience.

\section{RESULTS}

To analyze the data, we used a mixed measures three-way analysis of variance (ANOVA) with within-subject factors type of resolution (expected vs. unexpected) and pitch height (low vs. high) and between-subject factor music education (musicians vs. non-musicians).

First, we were interested in the effect of implicit learning of harmony. Specifically, we tested whether chord progressions with expected resolutions satisfy our participants more than chord progressions with unexpected resolutions. The results show that the type of resolution (expected vs. unexpected) significantly affected participants' subjective ratings of satisfaction, $F(1,95)=42,28, p<0,001, \eta_{\mathrm{g}}^{2}=0,051$, revealing that the participants were more satisfied with the expected chord progressions than with the unexpected ones (see Figure 2). 

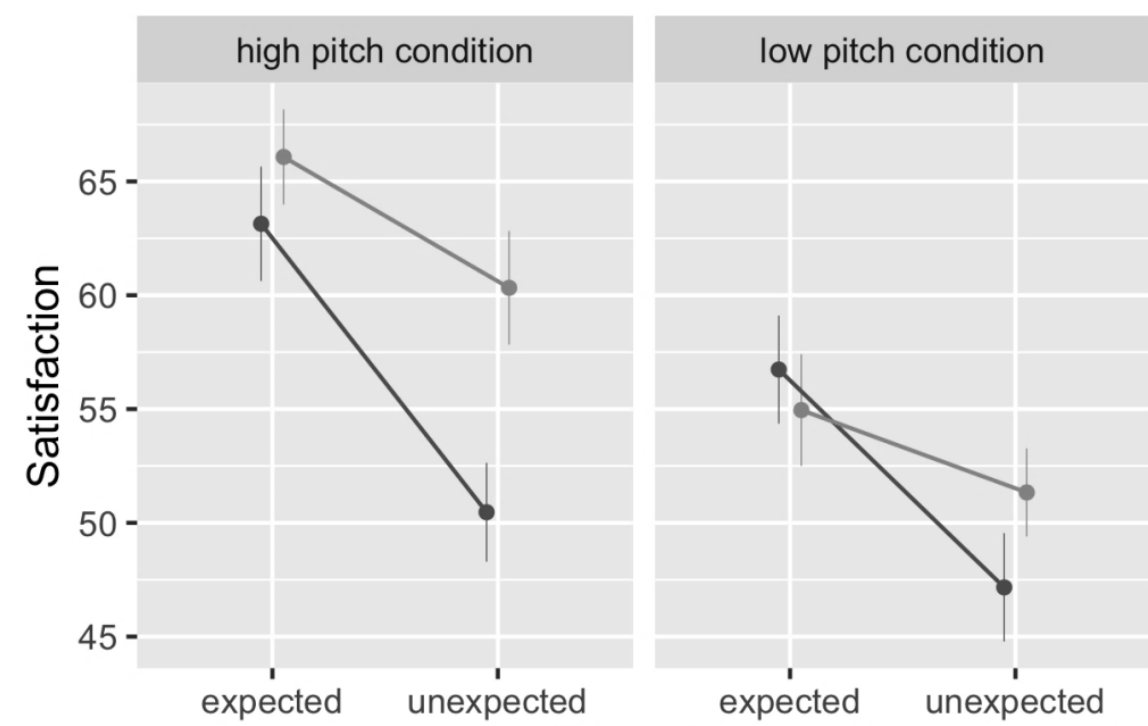

Education

musicians

non-musicians

\section{Resolution}

Figure 2. Subjective ratings of satisfaction with chord progressions in musicians and non-musicians depending on the type of resolution of harmonic progression and the progression's pitch condition. Error bars represent $95 \%$ Cousineau-Morey confidence intervals (CI) adjusted so that non-overlap of CIs corresponds to statistically significant difference as calculated with a mixed ANOVA [21].

Next, we were interested in whether musicians are in general more satisfied when listening to chord progressions than non-musicians, regardless of the resolution type. Results did not reveal a main effect of music education (musicians vs. non-musicians) on participants' subjective ratings of satisfaction with chord progressions, $F(1,95)=2,00, p=0,161, \eta_{\mathrm{g}}^{2}=0,013$. However, the results did show a significant interaction between music education and resolution type, $F(1,95)=7,61, p=0,007, \eta_{\mathrm{g}}^{2}=0,054$.

To identify which particular differences between pairs of means are significant, we performed additional post hoc tests with FDR-adjusted alpha levels. First, we compared musicians' and non-musicians' satisfaction rates separately for expected and unexpected resolutions. The results did not yield a significant main effect of music education in the expected resolution condition, $F(1,95)=0,04, p=0,841, \eta_{\mathrm{g}}^{2}<0,001$, however, it revealed a significant effect of music education in the unexpected resolution condition, $F(1,95)=5,59$, $p=0,0270, \eta^{2}=0,056$, showing that musicians are less satisfied with the unexpected resolutions than non-musicians. Next, we compared satisfaction rates in expected and unexpected conditions separately for musicians and non-musicians. The results revealed a significant effect of resolution in both musicians, $F(1,42)=28,36, p<0,001, \eta_{\mathrm{g}}^{2}=0,096$, and non-musicians $F(1,53)=13,93, p<0,001, \eta_{\mathrm{g}}^{2}=0,041$, reflecting higher satisfaction rates in expected vs. unexpected condition for both musicians and non-musicians.

Lastly, we tested whether the participants are more satisfied with chord progressions in the high pitch condition than in the low pitch condition. The results showed that the progression's pitch height significantly influenced participants' ratings of chord progression satisfaction, $F(1,95)=24,49, p<0,001, \eta_{\mathrm{g}}^{2}=0,010$, suggesting that the participants were more satisfied with the progressions in high pitch condition than with the progressions in low pitch condition. The interaction between the progression's pitch position and type of resolution was also significant, $F(1,95)=9,37, p=0,003, \eta_{\mathrm{g}}^{2}=0,002$, revealing a larger effect of type of resolution in high pitch position than in low pitch position (Figure 2). 
We carried out an additional exploratory analysis to test whether participants' satisfaction while listening to chord progressions might have also been affected by the specific sequence of the selected chord progression and whether this might have interacted with the resolution of progressions. We performed a two-way ANOVA with within-subject factors chord progression (nine typical chord progressions, see table 1) and type of resolution (expected vs. unexpected). The results showed a significant effect of the chord progression on the subjectively experienced satisfaction ratings, $F(8,768)=39,60, p<0,001, \eta_{\mathrm{g}}^{2}=0,021$, as well as significant interaction between type of resolution and type of progression, $F(8,768)=$ $3,50, p<0,001, \eta_{\mathrm{g}}^{2}=0,003$, suggesting that the extent of the effect of resolution type differed between different chord progressions.

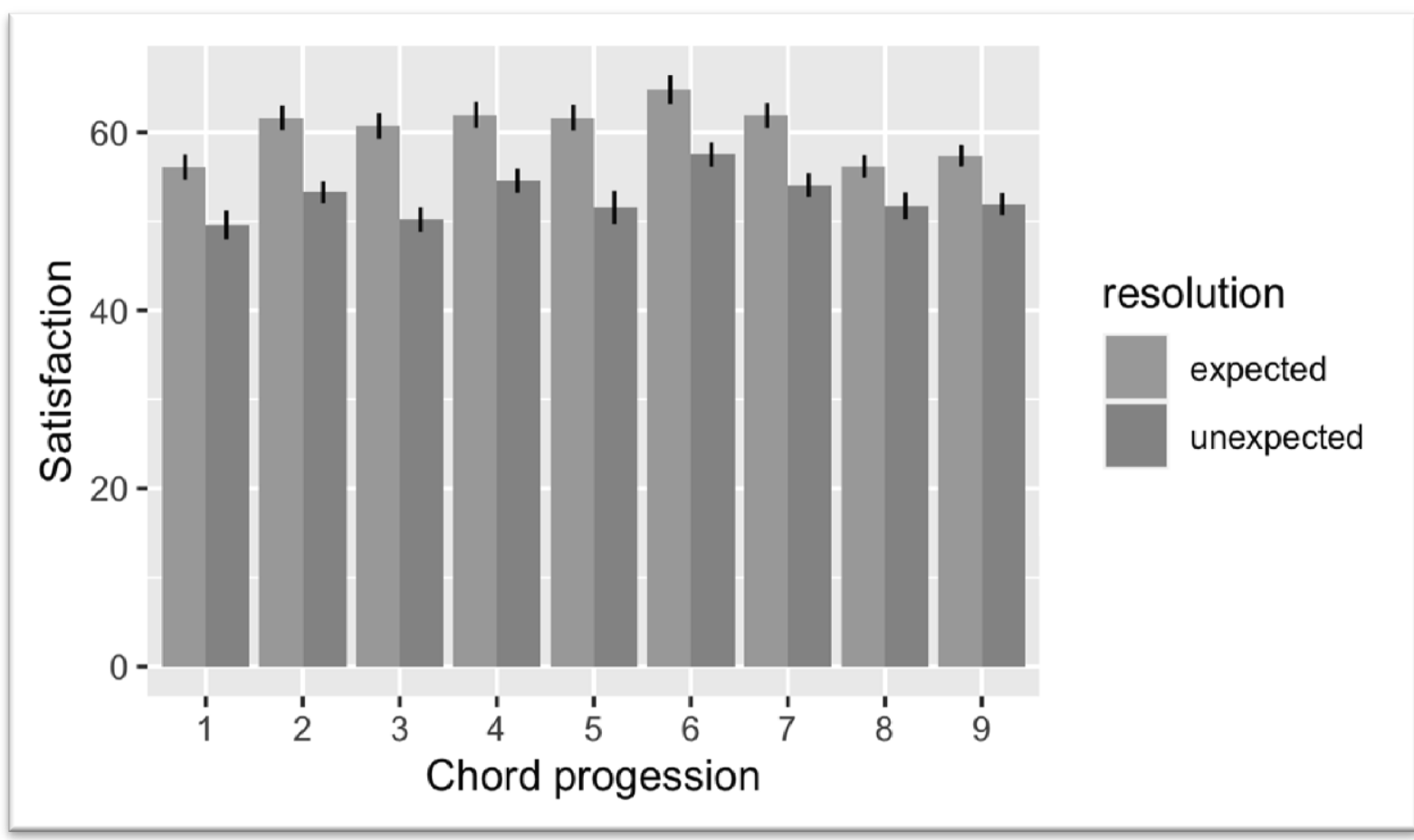

Figure 3. Subjective ratings of satisfaction concerning the nine types of harmonic progressions used (1 to 9) and the type of resolution. Error bars represent $95 \%$ Cousineau-Morey CIs adjusted so that non-overlap of CIs corresponds to statistically significant difference as calculated with a within-subject ANOVA [21].

\section{DISCUSSION}

Our study aimed to assess how implicit musical knowledge affects the experience of satisfaction while listening to expected and unexpected chord progressions and how this interacts with music education and the overall pitch height of chord progressions.

Our study's first goal was to evaluate the effect of implicit learning of harmony by comparing participants' satisfaction rates between expected and unexpected chord progressions and to assess whether this effect can be observed in both musicians and non-musicians. The results showed a significant effect of the type of resolution in both groups, reflecting higher satisfaction rates for expected vs. unexpected chord progressions. Our results suggest that both musicians and non-musicians implicitly learned the rules of harmony, which is consistent with previous research (e.g. $[7,8,15])$. Our study confirms that exposure to a particular type of music makes individuals internalize the music style and learn the rules of the harmony implicitly [2]. 
Next, we checked whether musicians and non-musicians differ in how they experience expected and unexpected resolutions in chord progressions. The satisfaction of musicians generally did not differ from the satisfaction of non-musicians, suggesting that participants, in general, experienced a similar degree of satisfaction while listening to the chord progressions. However, there was a significant interaction between the type of resolution and musical education. Post hoc analyses revealed a difference between musicians and non-musicians in how they experience satisfaction only in the unexpected chord progression condition, showing that the satisfaction in the unexpected resolution condition was smaller in musicians than in non-musicians. This contradicts previous findings indicating that musicians generally felt more pleasure while listening to both expected and unexpected chord progressions (e.g. [5]).

Results allow different interpretations. We can assume that musicians have attained better implicit knowledge based on their musical theory training compared to non-musicians [22]. Implicit knowledge can represent a sound basis for the development of long-term working memory, which enables musicians to recall the appropriate information from long-term memory faster and more efficiently than non-musicians [23]. This includes better recall of the typical chord progressions according to the western music style, as used in our study. Therefore, one explanation of why musicians' level of satisfaction in unexpected chord progressions was smaller than in non-musicians might be related to their superior implicit musical knowledge. The musicians possibly predicted the progressions to end on the tonic, and violations of the typical resolution in unexpected chord progressions had a larger negative effect, reflected in lower satisfaction rates than in non-musicians.

Another possible explanation of the difference in satisfaction of musicians and non-musicians while listening to unexpected resolutions might be related to how we assigned participants into two groups; musicians and non-musicians. In the first group, we included participants who have completed eight or more years of formal music education. That means that they have gained a lot of explicit, theoretical knowledge about western music theory. In the feedback provided after task completion, some musicians reported that they were disrupted because the chord progressions did not end on the tonic but on other scale levels. This suggests that musicians used not only their implicit but also their explicit knowledge to reason and rate their satisfaction level, emphasizing the role of declarative, long-term-explicit knowledge in shaping the experience of satisfaction. The role of implicit and explicit knowledge in shaping musical perception has previously also been recognized by other researchers [24].

Next, the present study results are consistent with the findings of Dellacherie et al. [10], who found that musicians respond more negatively to dissonances than non-musicians, whereas their response to consonances is similar. Dissonances as well as the unexpected resolutions, which were used in our study, can both be treated as tensions in western music, which can induce dissatisfaction during listening to music. Dellacherie et al. [10] suggest that a connection between dissonance and negative emotions is established because of long-term associative learning, possibly leading to a more pronounced effect of expectancy violations in musicians. Our results are also congruent with the findings of the EEG study of Pagès-Portabella and Toro [12], who found a larger early right-anterior negativity (ERAN) in response to listening to dissonance in musicians vs. non-musicians.

In summary, our results show that musical education modulates the processing of violations of typical chord progressions. Our next question was whether the chord progression's overall pitch height might also modulate the satisfaction while listening to expected and unexpected chord progressions. The results showed a significant main effect of pitch height on the reported levels of satisfaction, revealing that the participants rated the resolutions in high pitch height as more satisfactory than the resolutions in low pitch height. This finding is consistent with the low 
interval limit theory, which conjectures that the intervals in lower pitch positions sound muddy and unfocussed [14], possibly leading the participants to experience greater dissatisfaction while listening to low vs. high-pitch chord progressions.

Our results also showed a significant interaction between the type of resolution and pitch height, reflecting a larger effect of resolution type in the high-pitch condition. A possible explanation why the experience of listening to resolutions in the low pitch height was less affected by the type of resolution is that under low-pitch condition, resolutions were experienced as unfocused, which prevented the participants from discriminating between the resolution types in the first place. We can conclude that although a low interval limit is not an absolute limit and merely suggests the risk for the chord (or interval) to be experienced as unfocussed, our results show that the pitch height chosen for the low-pitch condition in our study did induce the experience of the dissonance and unclarity to at least some extent. This finding can help understand how the same harmonic pattern can evoke different experiences based on its pitch height.

\section{STUDY LIMITATIONS}

The present study has some potential limitations. First, we have noted a significant participant dropout. Of the 194 participants who initially began the test, only 105 completed the entire experiment. In their feedback, many participants reported having found the time necessary to complete the task too long. Additionally, many found the test itself boring. The dropout can therefore be attributed firstly to the duration of the experiment, which took approximately 30 minutes to complete, and secondly, to the type of chord progressions included in the task. The progressions mainly consisted of the same five chords that only varied in the sequence in which they appeared. This repetitiveness is why the participants could have found the task boring. We advise researchers conducting similar studies to use shorter chord presentation times to avoid the experiment being too time-consuming and present the participants with more diverse chord progressions.

Second, the additional exploratory analysis results revealed significant differences in participants' satisfaction between the selected chord progression sequences (i.e., participants preferred certain progressions types over others). Moreover, the effect of specific chord type significantly interacted with the resolution type of chord progressions, suggesting that the extent of the effect of resolution type differed between different chord progressions. This is an important finding that should be taken into account when designing future studies. Future studies should ensure that listeners experience a similar amount of satisfaction while listening to the selected chord progressions so that the studied effect of resolution type would not be confounded with other factors.

In addition to the participant dropout and the types of included progressions, we must also address the suitability of the criteria used to separate the participants into groups of musicians and non-musicians. Only those who reported having obtained at least eight years of formal education in music theory were assigned to a group of musically educated individuals, which resulted in a severely heterogeneous group of non-musicians. The latter included musically completely uneducated participants as well as individuals who have received several years of formal musical education. Despite not having been enrolled in as many years of music theory training as musicians, non-musicians could have had a comparable level of musical knowledge and experience as individuals with eight or more years of formal musical education. In this case, it would be more appropriate to include these individuals in the group of musicians. As the heterogeneity of the participants in the group of non-musicians could have had a potentially significant effect on the obtained results, we advise researchers conducting future studies to define their criteria for distinguishing the musically educated from the musically uneducated participants in a more elaborate and detailed way. We also suggest using music ability tests to objectively determine the participants' music perception skills, such as pitch discrimination. 


\section{CONCLUSIONS}

In the present study, we explored the effect of expectancy, music education, and pitch height on the satisfaction rates during listening to different chord progressions. As expected, the results showed that the participants were more satisfied with expected than unexpected chord progressions, confirming previous findings on the role of implicit learning of rules of harmony. Although results did not reveal an effect of music education during listening to expected chord progressions, musicians evaluated unexpected progressions as less satisfying than non-musicians, suggesting that musicians process music differently than non-musicians, and are more susceptible to violations of typical chord order. The results have also revealed that the difference in satisfaction between expected and unexpected progressions was larger in high-pitch vs. low-pitch condition, suggesting that chord progressions were more difficult to discriminate under low-pitch condition supporting the theory of low-interval limit. Though the results clearly show that the effect of resolution type is modulated by music education and the overall pitch height of music sequences, the findings need to be interpreted with caution due to methodological limitations.

\section{REFERENCES}

[1] Bharucha, J.J.: Event hierarchies, tonal hierarchies, and assimilation: A reply to Deutsch and Dowling.

Journal of Experimental Psychology: General 113(3), 421-425, 1984,

http://dx.doi.org/10.1037/0096-3445.113.3.421,

[2] Salimpoor, V.N.; Zald, D.H.; Zatorre, R.J.; Dagher, A. and McIntosh, A.R.: Predictions and the brain: how musical sounds become rewarding.

Trends in Cognitive Sciences 19, 86-91, 2015, http://dx.doi.org/10.1016/j.tics.2014.12.001,

[3] Piston, W.; Devoto, M. and Jannery, A.: Harmony. $3^{\text {rd }}$ edition.

W.W. Norton and Co., New York, 1987,

[4] Janata, P.: ERP measures assay the degree of expectancy violation of harmonic contexts in music.

Journal of Cognitive Neuroscience 7(2), 153-164, 1995,

http://dx.doi.org/10.1162/jocn.1995.7.2.153,

[5] Loui, P. and Wessel, D.: Harmonic expectation and affect in Western music: Effects of attention and training.

Perception and Psychophysics 69, 1084-1092, 2007,

http://dx.doi.org/10.3758/BF03193946,

[6] Bigand, E. and Pineau, M.: Global context effects on musical expectancy.

Perception and Psychophysics 59, 1098-1107, 1997,

http://dx.doi.org/10.3758/BF03205524,

[7] Bigand, E.; Madurell, F.; Tillmann, B. and Pineau, M.: Effect of global structure and temporal organization on chord processing.

Journal of Experimental Psychology: Human Perception and Performance 25(1), 184-197, 1999, http://dx.doi.org/10.1037/0096-1523.25.1.184,

[8] Koelsch, S.; Gunter, T.C.; Friederici, A.D. and Schröger, E.: Brain indices of music processing: 'Nonmusicians' are musical.

Journal of Cognitive Neuroscience 12, 520-541, 2000,

http://dx.doi.org/10.1162/089892900562183,

[9] Regnault, P.; Bigand, E. and Besson, M.: Different brain mechanisms mediate sensitivity to sensory consonance and harmonic context: evidence from auditory event-related brain potentials.

Journal of Cognitive Neuroscience 13(2), 241-255, 2001,

http://dx.doi.org/10.1162/089892901564298, 
[10] Dellacherie, D.; Roy, M.; Hugueville, L.; Peretz, I. and Samson, S.: The effect of musical experience on emotional self-reports and psychophysiological responses to dissonance. Psychophysiology 48(3), 337-349, 2011, http://dx.doi.org/10.1111/j.1469-8986.2010.01075.x,

[11]Parncutt, R. and Hair, G.: Consonance and dissonance in theory and psychology: Disentangling dissonant dichotomies.

Journal of Interdisciplinary Music Studies 5(2), 119-166, 2011, http://dx.doi.org/10.4407/jims.2011.11.002,

[12] Pagès-Portabella, C. and Toro, J.M.: Dissonant endings of chord progressions elicit a larger ERAN than ambiguous endings in musicians.

Psychophysiology 57(2), 2020, http://dx.doi.org/10.1111/psyp.13476,

[13] Corzine, V.: Arranging music for the real world: classical and commercial aspects. Mel Bay, St. Louis, 2002,

[14] Hoffmann, R.: Low interval limits. http://www.robin-hoffmann.com/dfsb/low-interval-limits, accessed June $5^{\text {th }} 2020$,

[15] Tillmann, B.; Bharucha, J.J. and Bigand, E.: Implicit learning of tonality: A self-organizing approach.

Psychological Review 107(4), 885-913, 2000,

http://dx.doi.org/10.1037/0033-295X.107.4.885,

[16] Apple Inc.: Apple Inc. Logic Pro X, ver. 10.4.4. http://apps.apple.com/us/app/logic-pro/id634148309? $\mathrm{mt}=12$, accessed $12^{\text {th }}$ June 2020,

[17] Native instruments: The Gentleman. http://www.native-instruments.com/en/products/komplete/keys/the-gentleman, accessed $12^{\text {th }}$ June 2020,

[18] Native Instruments: Kontakt 6 Player, ver. 6.2.1.

http://www.native-instruments.com/en/products/komplete/samplers/kontakt-6-player, accessed $12^{\text {th }}$ June 2020 ,

[19] Stoet, G.: PsyToolkit - A software package for programming psychological experiments using Linux.

Behavior Research Methods 42(4), 1096-1104, 2010, http://dx.doi.org/10.3758/BRM.42.4.1096,

[20] Stoet, G.: PsyToolkit - a novel web-based method for running online questionnaires and reaction-time experiments.

Teaching of Psychology 44(1), 24-31, 2017, http://dx.doi.org/10.1177/0098628316677643,

[21]Baguley, T.: Calculating and graphing within-subject confidence intervals for ANOVA. Behavior Research Methods 44(1), 158-175, 2012, http://dx.doi.org/10.3758/s13428-011-0123-7,

[22] Thorpe, L.; Cousins, M. and Bramwell, R.: Implicit knowledge and memory for musical stimuli in musicians and non-musicians. Psychology of Music 48(6), 836-845, 2020, http://dx.doi.org/10.1177/0305735619833456,

[23] Ericsson, K.A. and Kintsch, W.: Long-term working memory. Psychological Review 102(2), 211-245, 1995, http://dx.doi.org/10.1037/0033-295X.102.2.211,

[24] Scherer, K.R. and Coutinho, E.: How music creates emotion: A multifactorial process approach.

In: Cochrane, T.; Fantini, B. and Scherer, K.R., eds.: Series in affective science. The emotional power of music: Multidisciplinary perspectives on musical arousal, expression, and social control. Oxford University Press, Oxford, pp.121-145, 2013, http://dx.doi.org/10.1093/acprof:oso/9780199654888.003.0010. 